> Les perturbateurs endocriniens (PE) ont été définis soit comme étant des substances étrangères à l'organisme qui induisent des effets délétères sur cet organisme ou sur sa descendance en raison d'une altération de la fonction endocrine, soit comme étant des agents exogènes qui interfèrent avec la production, la libération, le transport, le métabolisme, la liaison, l'action ou l'élimination des ligands naturels responsables du maintien de l'homéostasie et de la régulation du développement de l'organisme. Ces deux définitions indiquent que les effets induits par les $P E$ sont susceptibles de mettre en jeu des mécanismes relevant, à un niveau ou à un autre, de l'homéostasie hormonale. Hormis le lien clairement établi entre le diéthylstilbestrol, les anomalies de la reproduction et les cancers, il y a très peu de certitudes quant à l'impact des $P \varepsilon$ sur la santé humaine. En raison du peu de données disponibles, l'implication éventuelle des PE dans l'augmentation de l'incidence du cancer du sein, voire de l'endométriose, et de pubertés précoces chez la fillette reste hypothétique. En revanche, la détérioration de la santé reproductive masculine est au centre des préoccupations relevant des relations $P \mathcal{E}$-santé humaine. Cette revue bibliographique vise à faire l'état des lieux de la question de la perturbation endocrinienne en mettant plus particulièrement l'accent sur la problématique des contaminants alimentaires. <

Le système endocrinien relève d'un mode complexe de communication et de régulation entre un messager chimique endogène et les cibles sur lesquelles il peut agir afin de modifier certaines fonctions de l'organisme. Toute substance exogène susceptible d'altérer ce fonctionnement est appelée perturbateur endocrinien $(P E)$.

Article reçu le 10 mars 2006, accepté le 25 septembre 2006.

\section{Le concept de perturbation endocrinienne et la santé humaine}

\author{
Jean-Pierre Cravedi, Daniel Zalko, \\ Jean-François Savouret, Arnaud Menuet, \\ Bernard Jégou
}

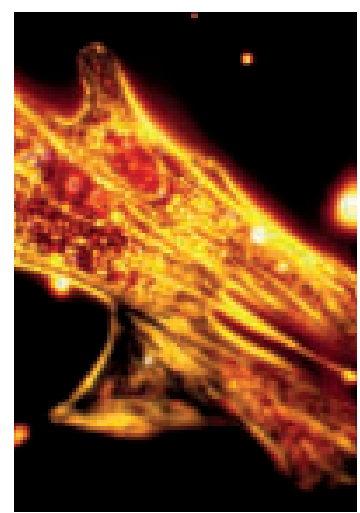

\author{
J.P. Cravedi, D. Zalko: \\ Inra, UMR 1089, \\ Xénobiotiques, BP 3, \\ 31931 Toulouse Cedex 9, France. \\ J.F. Savouret : Inserm UMR-747, \\ Université Paris Descartes, \\ 45, rue des Saints-Pères, \\ 75006 Paris, France. \\ A. Menuet, B. Jégou : \\ Inserm U625, \\ Université Rennes 1, \\ GERHM, IFR 140, \\ Campus Beaulieu,
}

En Europe, un PE a été Rennes, 35042 France.

défini comme étant bernard.jegou@rennes.inserm.fr une substance étran-

gère à l'organisme qui produit des effets délétères sur l'organisme ou sur sa descendance, à la suite d'une modification de la fonction hormonale ${ }^{1}$. Pour l'Agence américaine de l'environnement ( $(P A)$, un $P \varepsilon$ est un agent exogène qui interfère avec la production, la libération, le transport, le métabolisme, la liaison, l'action ou l'élimination des ligands naturels responsables du maintien de l'homéostasie et de la régulation du développement de l'organisme [1]. On notera que la définition américaine passe sous silence les effets directs des xénobiotiques passant par des voies spécifiques ainsi que les effets transgénérationnels.

Quoi qu'il en soit, la singularité du concept de perturbation endocrinienne apparaît dans ces deux définitions: les effets observés peuvent mettre en jeu les mécanismes de signalisation, de régulation et d'action physiologiques plutôt que les mécanismes classiques de la toxicité. II existe un faisceau croissant d'indications suggérant l'impact de substances chimiques sur les régulations endocrines, à commencer par celle des hormones stéroïdiennes qui interviennent sur le

${ }^{1}$ European Workshop on the Impact of Endocrine Disrupters on Human Health and Wildlife, Weybridge, Royaume-Uni, 1996, European Union Report EUR17459. 
développement et la fonction de reproduction. Les évidences sont nombreuses en matière d'écotoxicologie et d'impact sur la faune sauvage, principalement aquatique. En effet, plusieurs études ont établi un lien dans les populations sauvages entre la présence de polluants, connus en laboratoire pour mimer l'action d'hormones stéroïdiennes, et la survenue d'anomalies du développement et de la reproduction des alligators, des gastéropodes, des phoques, de certains poissons et des batraciens [2].

Les liens de causalité entre xénobiotiques et perturbation endocrinienne chez les humains sont plus rares. La tragédie du diéthylstilbestrol (DES) est pourtant là pour rappeler qu'une substance chimique se comportant comme une hormone est capable non seulement d'affecter gravement la santé des personnes exposées, mais également d'entraîner des troubles irréversibles dans leur descendance (Figure l). Le cas du DES révèle que : le placenta humain n'est pas une barrière impénétrable aux xénobiotiques; l'exposition de la mère peut avoir des effets inattendus sur la progéniture, même plusieurs décennies plus tard; l'exposition aux PE peut avoir des conséquences graves sur la santé, incluant la survenue de cancers [2].

Hormis ce lien clairement établi entre DES et cancers, il y a très peu de certitudes quant à l'impact des PE sur la santé humaine. Cependant, plusieurs résultats convergents obtenus sur des espèces de laboratoire et/ou à partir d'études épidémiologiques étayent l'hypothèse selon laquelle des troubles de la reproduction, du fonctionnement thyroïdien ou du neuro-comportement pourraient résulter d'expositions à des molécules chimiques induisant des réponses positives dans les tests in vitro et/ou in vivo permettant de détecter les $P E$. En raison du peu de données disponibles, les interrogations actuelles, qui portent sur l'implication éventuelle des PE dans l'augmentation de l'incidence du cancer du sein, voire de l'endométriose et de pubertés précoces chez la fillette, restent généralement sans réponse. En revanche, la possible détérioration de la santé reproductive masculine figure au premier plan des préoccupations et des avancées corrélant exposition aux $P \varepsilon$ et santé humaine. Cette détérioration repose sur un triptyque associant la dégradation de la qualité du sperme, l'augmentation de l'incidence du cancer du testicule et l'augmentation d'anomalies du

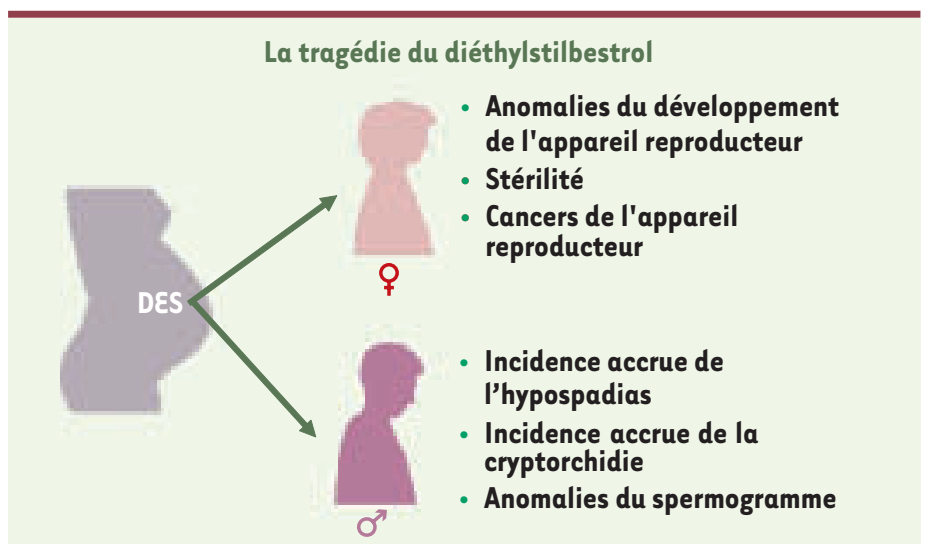

Figure 1. Résumé des conséquences sur la descendance féminine (ㅇ) et masculine ( 6 ) du traitement des femmes enceintes par le diéthylstilbestrol (DES). tractus génital masculin [2, 3]. Les controverses autour de cette question portent sur la réalité de la baisse des paramètres séminaux compte tenu, par exemple, de la variabilité importante des méthodes d'analyse de ces paramètres [4], et sur la réalité également de l'augmentation de l'incidence de la cryptorchidie et de l'hypospadias (non fermeture de l'urètre nécessitant une correction chirurgicale au niveau de pénis), d'aucuns signalant que l'attention portée par les médecins à ces anomalies est plus importante aujourd'hui qu'elle ne l'était autrefois, ce qui fausserait les statistiques. Toutefois, une lecture critique de la littérature permet de considérer qu'il existe effectivement une forte présomption de diminution séculaire de la concentration spermatique à certains endroits du monde (dont la région parisienne) [5, 6], que l'incidence de l'hypospadias augmente probablement alors que l'augmentation de celle du cancer du testicule est avérée partout dans le monde (France incluse), là où les épidémiologistes ont eu accès à des registres des cancers.

Cette situation a conduit Shakkebaek et al. à émettre I'hypothèse selon laquelle les éléments sus-cités du triptyque relatifs à la santé reproductive masculine sont impliqués au travers du concept de syndrome de dysgénésie testiculaire (SDT) qui les considère comme relevant d'une même entité pathologique pouvant avoir des causes communes dont une ou des causes environnementales [7]. Les PE identifiés se présentant essentiellement comme des analogues ou des antagonistes des hormones stéroïdiennes sexuelles, le rapprochement a été effectué entre les concepts de SDT et de perturbation endocrinienne. Toutefois, les preuves expérimentales qui pourraient effectivement établir la réalité du SDT sont encore fragiles.

Chacune des trois composantes de l'hypothèse du SDT, prise séparément, étant susceptible d'altérer la reproduction masculine, il est légitime de penser que la fertilité des couples a pu décliner depuis plusieurs décennies. Cependant, ce n'est pas l'augmentation du recours à la procréation médicalement assistée (PMA) observée ces dernières décennies qui peut permettre d'étayer cette hypothèse car, l'offre de PMA médicale s'étant accrue, il est logique que la demande des patients se soit également accrue. En outre, il est actuellement impossible de vérifier l'évolution séculaire de la fertilité humaine car les paramètres spermatiques qui ont été étudiés rétrospectivement (concentration, mobilité, morphologie) ne sont pas les plus sensibles pour évaluer la «fécondance» des hommes. En outre, les données les plus fiables pour évaluer l'évolution de la «fécondance » humaine, c'est-à-dire les valeurs du délai nécessaire à concevoir (DNC), ne sont pas dispo- 
nibles [8]. À noter qu'une étude rétrospective récente parmi les jumeaux danois n'a pas mis en évidence de tendance à la diminution de la probabilité de grossesse [9]. De façon intéressante, il apparaît qu'il existe des différences géographiques importantes des paramètres de la santé reproductive masculine, ce qui est maintenant bien établi par toute une série d'études récentes [10]. Le nombre relativement important de facteurs susceptibles d'expliquer ces variations géographiques génétiques, nutritifs, psychosociaux, environnementaux - ne permet pas vraiment d'en privilégier un en particulier pour le moment [8].

La majeure partie des PE décrits sont des substances ayant des effets œstrogéniques, ce qui comprend les molécules capables de mimer l'effet du $17 \beta$-œstradiol, mais n'est pas limité à celles-ci (Tableau I). Les structures chimiques impliquées sont parfois très différentes de celle de l'œstradiol, ce qui signifie qu'il n'est pas possible d'identifier un PE sur la base de sa structure chimique. II faut par ailleurs noter que, parmi les $P E$, figurent des substances d'origine naturelle tels que les phyto-œstrogènes présents dans le soja (Tableau I). II est donc nécessaire de procéder à des tests in vivo et in vitro pour mesurer l'activité œstrogénique ou éventuellement anti-œstrogénique.

Même si les PE, dont l'activité biologique passe par une interaction avec les récepteurs des hormones sexuelles, sont à l'heure actuelle les mieux connus, il convient de

\begin{tabular}{lcc}
\hline $\begin{array}{l}\text { Type d'activité } \\
\text { hormonale }\end{array}$ & Analogue & Antagoniste \\
Cistrogène & $+++a$ & $++a$ \\
Androgène & $++b$ & $++++c$ \\
Glucocorticoïde & - & $? d$ \\
Thyroïdienne & $?$ e & $?$ \\
\hline
\end{tabular}

Tableau I. Exemples d'activités xénohormonales décrites dans la littérature. ++++ nombreux produits; ++ : quelques produits; ?: activité présomptive. a : œstradiol (excrétats humains et animaux), bisphénol A, DES, DDT (pesticide), nonylphénol (agent tensio-actif), éthinyl-œstradiol (pilule féminine), phyto-œstrogènes. $b$ : l'androstènedione est un androgène qui peut être présent dans les cours d'eau en aval d'usines de pâte à papier. II est issu de la métabolisation par Mycobacterium smegmatis d'un phytostérol, le stigmastérol. L'androstènedione peut être responsable de la masculinisation d'un poisson comme la gambusie. $c$ : phtalates. $d$ : I'arsenic pourrait bloquer la voie de signalisation des glucocorticoïdes via leur récepteur. e : les PCB (polychlorobiphényles) sont susceptibles d'induire une action analogue à celle des hormones thyroïdiennes mais sans se lier à leurs récepteurs. prendre en compte les multiples régulations du système neuro-endocrinien pour pouvoir estimer le risque encouru. II est physiologiquement cohérent que des dysfonctionnements thyroïdiens ou surrénaliens puissent induire des modifications des régulations des hormones de la reproduction. Les conséquences possibles d'une perturbation de l'homéostasie par des molécules chimiques présentes dans l'environnement de l'homme ne se limitent donc pas à des effets potentiels sur la reproduction. II apparaît de plus en plus évident que les périodes pré- et post-natales constituent des fenêtres critiques d'exposition, pouvant expliquer des effets délétères des PE observés à partir de la puberté. La sensibilité de l'embryon et du fœus à des $P E$ susceptibles de traverser la barrière placentaire est mise en avant

- Hormones mammifères naturelles (œstrogènes)

- Substances naturelles/phytohormones (génistéine, daïdzéine, coumestrol, zéaralénone...)

- Produits phyto-sanitaires (herbicides, fongicides, insecticides, nématocides)

- Polluants de l'industrie chimique (alkylphénols, bisphénol A, phthalates, tributylétain, métaux lourds...)

- Polluants pharmaceutiques/vétérinaires (DES, promoteurs de croissance...)

Tableau II. Différentes catégories de perturbateurs endocriniens.

- Stéroïdes

Estrogènes (lait, œufs...)

- Produits pharmaceutiques

Diéthylstilbestrol (viande, eau)

Éthinyl-œstradiol (eau)

Androgènes (compléments alimentaires...)

Phyto-œstrogènes (lait et autres préparations à base de soja)

- Produits végétaux

Zéaralénone (céréales)

Génistéine/isoflavone, daïdzéine (soja...)

Coumestrol (haricots...)

- Produits industriels

Nonylphénol (eau)

Bisphénol A (boissons, aliments divers)

Tableau III. Exemples de composés œstrogéniques présents naturellement dans des aliments ou à la suite de diverses contaminations. Les contaminations sont consécutives au traitement d'animaux d'élevage par des inducteurs de croissance (DES) ; au dopage (compléments alimentaires) et à la prescription de préparations pharmaceutiques à base de soja; à l'excrétion naturelle animale ou humaine (œstrogènes, éthinyl-œstradiol, DES...) ; au développement sur des aliments de moisissures contenant un myco-œstrogène tel que le zéaralenone; à l'évacuation de détergents dans les égouts (nonylphénol...) ; ou encore au relargage de molécules contenues dans les matières plastiques (phthalates, bisphénol A). 
pour expliquer la toxicité avérée ou supposée de nombreux xénobiotiques. Les sources d'exposition aux PE comme la nature des PE sont multiples. Les $P \varepsilon$ peuvent être présents dans les différents compartiments de l'environnement (aérien, aquatique, terrestre), mais également dans les aliments (d'origine animale ou végétale) ; ils peuvent provenir des emballages alimentaires, des produits de combustion, des traitements phytosanitaires, des détergents et de l'industrie chimique en général. À ces substances d'origine anthropogénique s'ajoutent certains micronutriments que l'on trouve principalement dans les végétaux (Tableau II). Nous privilégierons ici les contaminants alimentaires, c'est-à-dire les résidus de xénobiotiques présents dans les denrées, les migrats d'emballage et éventuellement ceux susceptibles de se former au cours des processus de préparation des aliments; seront également traités les microconstituants alimentaires ayant des propriétés hormonomimétiques (Tableau III).

\section{Polluants organiques persistants (POP)}

Les POP sont des substances qui posent des problèmes particuliers en matière de risque environnemental ou alimentaire. Outre le fait qu'il s'agit de composés toxiques, leur persistance, leur caractère lipophile et leur capacité à s'accumuler dans les différents maillons de la chaîne alimentaire peuvent se traduire par des niveaux de concentration élevés dans certains aliments tels que les poissons gras. Sont classés parmi les POP des produits industriels comme les PCB (polychlorobyphényles), des pesticides comme les insecticides organochlorés (DDT, lindane, aldrine, toxaphène) ou encore les dioxines et les furanes qui sont des contaminants des produits organohalogénés ou qui se forment au cours de la combustion de matières organiques en présence de chlore. Aujourd'hui, la fabrication ou la commercialisation de ces substances indésirables est interdite ou très réglementée dans le monde occidental. Néanmoins, leur grande résistance à la dégradation biotique ou abiotique et l'utilisation de pesticides organochlorés dans certains pays tropicaux ou subtropicaux expliquent qu'ils sont toujours détectés dans les denrées, même si les concentrations sont globalement en constante diminution depuis les 20 dernières années.

L'exemple des PCB illustre particulièrement bien la complexité du problème de la perturbation endocrinienne, en particulier lorsque plusieurs cibles sont touchées et plusieurs mécanismes d'action mis en œuvre. Outre leur impact sur le développement ou la reproduction, les PCB dont la structure moléculaire globale n'est pas coplanaire peuvent perturber le fonctionnement de la thyroïde et modifier le niveau circulant des hormones thyroïdiennes [11]. Plusieurs auteurs ont pu montrer que ces perturbations, tant au plan du développement que de la fonction thyroïdienne, étaient souvent le fait de métabolites hydroxylés des PCB [12]. D'autres travaux ont démontré que l'effet des PCB ou de leurs métabolites pouvait également provenir d'une inhibition de la sulfotransférase prenant en charge la conjugaison de l'œstradiol [13] ou des hormones thyroïdiennes.

Enfin, les PCB dont la structure est coplanaire interagissent avec le récepteur des arylhydrocarbures (AhR) comme les dioxines et certains hydrocarbures polycycliques et/ou halogénés comme le benzoa-pyrène de la fumée de tabac et la 3-nitrobenzanthrone des gaz d'échappement de moteurs diesel. II s'agit là d'un effet perturbateur original car ce récepteur ne possède pas de ligand connu et ne semble pas impliqué dans la physiologie hormonale stricto sensu mais plutôt dans l'induction des cytochromes des voies de détoxification.

Les dioxines ont fait l'objet de travaux au moins aussi nombreux que les $P C B$ en matière de perturbations endocriniennes. Un rapport récent de I'IPCS (International programme on chemical safety, 2001 ; global assessment of the state-of-the-science of endocrine disruptors, http://ehp.niehs.nih.gov/who) sur ce sujet mentionne une baisse de la quantité de sperme et du rapport testostérone/hormone gonadotrope ainsi qu'une augmentation significative de LH (luteinizing hormone) et de FSH (follicle stimulating hormone) chez des salariés anciennement exposés aux dioxines. Une modification du sex ratio (excès de filles) a également été notée à Seveso depuis la catastrophe en 1976 [14]. Comme les PCB, les dioxines sont également susceptibles de modifier la clairance de l'hormone thyroïdienne T4 et d'affaiblir les défenses immunitaires en agissant sur les lymphocytes T [15]. D'autres contaminants lipophiles et persistants tels que les retardateurs de flamme polybromés (polybromodiphényléthers) sont également classés parmi les PE et font l'objet de plusieurs programmes de recherches européens en raison, notamment, de leur impact sur la fonction thyroïdienne.

\section{Constituants des plastiques}

\section{4-nonylphénol}

Dans l'environnement, ce composé provient, pour partie, de la dégradation de détergents non ioniques tels que les alkylphénols polyéthoxylates. II intervient également dans la composition de nombreuses matières plastiques à partir desquelles il peut être relargué dans l'eau. Tous les résultats publiés dans la littérature démontrent que le nonylphénol est capable de se fixer sur le récepteur de l'œstradiol et d'avoir un effet œstrogénique, y compris chez les mammifères. En dépit du fait que le nonylphénol est 1000 à 10000 fois moins œstrogénique que l'œstradiol, selon les modèles biologiques utilisés, plusieurs travaux expérimentaux indiquent des atteintes de la fertilité, de la reproduction et du développement. D’autres alkylphénols tels que le 4-tert-octylphénol ont des propriétés analogues au nonylphénol. Dans une étude récente menée chez la souris, Acevedo et al. [16] ont montré que le nonylphénol augmentait significativement le taux de cancer mammaire chez des animaux ayant reçu pendant 32 semaines une dose quotidienne de $30 \mathrm{mg} / \mathrm{kg}$ de nonylphénol administrée par voie orale [16]. 


\section{Bisphénol A (BPA)}

II s'agit d'un produit industriel utilisé comme monomère dans la synthèse des matières plastiques et des résines époxy. II est présent dans les revêtements de boîtes de conserve, certains biberons, les résines d'obturation utilisées en dentisterie. Environ trois millions de tonnes de BPA sont produites chaque année dans le monde. Un article récent [17] indique que $95 \%$ des échantillons d'urine collectés auprès de 394 adultes aux États-Unis contenaient des niveaux quantifiables de BPA. Comme le nonylphénol, le bisphénol $A$ est un œstrogéno-mimétique capable de se lier au récepteur $\alpha$ des œstrogènes et son action est environ 1000 fois inférieure à celle de l'œstradiol. D'après les travaux récents d'A. Soto et al. chez la souris CDl, une exposition in utero à des doses faibles provoque chez les mâles une augmentation du poids de la prostate et une diminution du poids de l'épididyme, alors que chez les femelles apparaissent des altérations du développement des glandes mammaires [18]. Récemment, Timms et al. ont démontré chez des souris gravides exposées à des doses de $10 \mu \mathrm{g} / \mathrm{kg} / \mathrm{j}$, c'est-à-dire proches des valeurs d'exposition de la femme enceinte, des modifications du développement de la prostate chez les fœtus qui laissent présager un risque de cancer accru ultérieurement chez l'adulte [19]. Signalons que chez la souris, le passage transplacentaire du BPA (4\% d'une dose de $20 \mu \mathrm{g} / \mathrm{kg}$ ) est avéré [20].

\section{Phtalates}

Les phtalates sont des dérivés diesters de l'acide phtalique. Parmi les plus fréquemment utilisés aujourd'hui figurent les phtalates de di-n-butyle, de butylbenzyle, de di-n-octyle, de di-isononyle, de di-isodécyle, de di-(2éthylhexyle). Ces composés interviennent dans la fabrication et la composition d'un grand nombre de matériaux et produits tels que chlorure de polyvinyle (PVC), polyéthylène téraphtalate (PET), jouets, peintures industrielles, adhésifs et colles, encres, revêtements de sol en vinyle, huiles lubrifiantes, matériels électriques, détergents, solvants, produits pharmaceutiques et cosmétiques, emballages plastiques, etc. La consommation des phtalates en Europe est d'environ un million de tonnes par an [21]. Ils sont très répandus dans l'environnement et l'on peut également les trouver dans les aliments, après leur migration à partir d'emballages. Le phtalate de di-n-butyle, le phtalate de butylbenzyle et le phtalate de di-(2-éthylhexyle) peuvent provoquer, via une action anti-androgénique, une atteinte de l'appareil reproducteur mâle (au cours du développement et au stade adulte) et une toxicité embryo-fœtale pouvant conduire à une augmentation de la mortalité intra-utérine ou à une diminution du taux de survie postnatale $[22,23]$. En se liant au récepteur des androgènes, les phtalates bloquent le fonctionnement normal de ces hormones, sans pour autant activer ces récepteurs (effet antagoniste). Ils sont également faiblement œstrogéniques [24]. Des études épidémiologiques ont également mis en évidence une corrélation entre, d'une part le développement prématuré des seins et la présence de résidus de phtalates dans le sérum des jeunes filles portoricaines [25] et, d'autre part, l'exposition aux phtalates chez les mères et la distance anogénitale chez les nouveau-nés [6]. Toutefois, ces dernières données mériteraient d'être confirmées.

\section{Pesticides}

Plusieurs pesticides, parmi lesquels des insecticides (DDT, endosulfan, dieldrine, méthoxychlore, dicofol, chlordécone, toxaphène), des nématocides (aldicarbe), des herbicides (alachlore, atrazine, nitrofène, 2,4D), des fungicides (bénomyl, mancozeb, vinchlozoline) figurent sur les listes de perturbateurs endocriniens. Certaines de ces molécules actives sont aujourd'hui interdites en Europe mais peuvent néanmoins toujours être présentes dans notre environnement. De nombreuses études ont recherché une association éventuelle entre l'exposition à certains pesticides et leurs effets sur la reproduction, en particulier la fertilité masculine. Les plus probantes sont celles qui concernent le 1,3-dibromo-3-chloropropane (DBCP) et le chlordécone. Le premier est un nématocide qui n'est plus autorisé, mais dont l'utilisation chez des ouvriers de bananeraies a entraîné une très forte altération de la qualité du sperme, une baisse de la fertilité, une augmentation du taux de FSH et du rapport testostérone/ hormones gonadotropes, ces anomalies étant souvent irréversibles. Toutefois, le caractère intrinsèque de $P \mathcal{E}$ du $D B C P$ n'est pas établi. En revanche, une baisse significative du nombre de spermatozoïdes et de leur mobilité a été observée chez des ouvriers ou des rats exposés au chlorodécone [26], celui-ci s'avérant un xéno-œstrogène clairement démontré [27].

Nous ne pouvons exposer ici la totalité des pesticides suspectés d'être des $P \varepsilon$, mais l'exemple de la vinchlozoline mérite également d'être cité. II s'agit d'un fongicide dont deux métabolites ont des propriétés anti-androgéniques pouvant entraîner des perturbations de la reproduction chez le rat [28]. Très récemment, Anway et al. ont découvert que l'exposition à la vinchlozoline de rattes, entre le $8^{\mathrm{e}}$ et le $15^{\mathrm{e}}$ jour de gestation, avait des effets non seulement chez les animaux exposés in utero ( $F 1)$, mais également sur ceux des générations ultérieures (F2, F3, F4) [29]. Ces effets ne font pas intervenir de modification de séquence de l'ADN. Ils obéiraient plutôt à une transmission épigénétique [29]. Il faut toutefois observer ici que ces résultats font l'objet d'une controverse et devront donc être confirmés par une autre équipe.

\section{Phyto-œstrogènes}

Les aliments à base de soja peuvent contenir des teneurs importantes en phyto-œstrogènes ( $P O)$, principalement de la daïdzéine et de la génistéine. Les recherches sur les PO ont connu un essor important à partir des années 1990 lorsque certaines études épidémiologiques ont tenté de corréler la consommation de ces produits chez les populations asiatiques avec la fréquence moins élevée, en comparaison des populations occidentales, de certains types de cancers ou de certaines maladies cardiovasculaires. Par ailleurs, les PO pourraient également jouer un rôle 
protecteur vis-à-vis de l'ostéoporose et de certains troubles associés à la ménopause [30]. Cependant, plusieurs de ces résultats font l'objet de controverses et quelques auteurs ont souligné l'absence d'études complètes de toxicité des $\mathrm{PO}$, sans lesquelles il ne peut être établi de réelle analyse risques/bénéfices de tels composés pour l'homme.

Les niveaux mesurés dans le lait de soja peuvent dépasser 200 mg/l [31] et plusieurs préparations à base de soja atteignent des teneurs supérieures à $500 \mathrm{mg} / \mathrm{kg}$ [32]. Ces aliments conduisent à des ingestions quotidiennes qui sont de l'ordre de 30 à $50 \mathrm{mg}$ d'isoflavones, c'est-à-dire identiques à celles des préparations préconisées chez les femmes ménopausées.

Plusieurs scientifiques se sont inquiétés des risques pour le nourrisson d'une consommation excessive en PO. Les laits de soja pour nourrissons ont des concentrations qui varient de 32 à $47 \mathrm{mg} /$ l ce qui se traduit par une exposition des nourrissons de 22 à $45 \mathrm{mg}$ d'isoflavones par jour (6-11 mg/kg/j) [33]. L'exposition de nourrissons ou de jeunes enfants à des quantités élevées de PO pourrait entraîner des effets indésirables graves tels que des dysfonctionnements de la thyroïde, du système immunitaire ou encore de la régulation hormonale.

Quelques travaux expérimentaux viennent étayer ces craintes en montrant une augmentation du taux de cancers mammaires chez des rats exposés in utero à la génistéine [34], l'induction d'adénocarcinomes de l'utérus chez des souris traitées pendant les cinq premiers jours de la vie par $50 \mathrm{mg}$ de génistéine $/ \mathrm{kg} / \mathrm{j}$ [35], un lien entre régime riche en PO au cours de l'enfance et apparition d'un diabète insulino-dépendant [36], et le nombre apparemment plus élevé d'enfants atteints d'hypospadias chez les mères végétariennes [37].

\section{Conclusions et perspectives}

Le fait que le système endocrinien soit indispensable au maintien des équilibres biologiques nécessaires à la vie explique que l'on s'interroge beaucoup aujourd'hui sur les conséquences d'une éventuelle rupture de cet équilibre par les contaminants de l'environnement. Hormis le cas du DES, des pesticides comme le DDT ou le chlordécone, connus depuis longtemps comme facteurs d'infertilité chez l'homme, et dont le mode d'action relève bien (DES) ou probablement (pesticides ci-dessus) d'une perturbation endocrinienne, il existe peu de données fiables établissant un lien entre $P \varepsilon$ et santé humaine. II faut en outre noter que même pour des produits tels que le DES et les pesticides cités, il s'agit de situations d'exposition difficilement extrapolables à l'ensemble de la population. Néanmoins, la problématique des faibles doses, telle qu'elle apparaît aujourd'hui avec le bisphénol $A$, ou le fait que certains mélanges de $P E$ soient toxiques alors que pris individuellement et aux mêmes doses, chacun des composés ne l'est pas, incite à la vigilance. Par ailleurs, la liste des substances suspectées d'être des $P E$ est bien plus longue que les quelques exemples que nous avons brièvement traités. Ainsi, à partir d'une liste initiale de 564 composés suspectés d'être des PE [38], la Commission Européenne en a retenu prioritairement 66, parmi lesquels figurent, outre les quelques familles citées ci-dessus, des métaux et organométalliques, des composés bromés et des dérivés phénoliques.

La présence de xénobiotiques et de composés pharmaceutiques dans l'environnement fait l'objet d'actions européennes depuis le $5^{e}$ programme commun de recherche et développement (PCRD). Celuici comprend un groupe de quatre grands programmes dénommé CREDO (www.credocluster.info/intro.htm). Le $6^{\mathrm{e}}$ PCRD prolonge ces actions (www.cordis.lu/food/home.html) en y adjoignant divers programmes tels que des STREP à visée plus appliquée. Un réseau d'excellence (CASCADE, www.cascadenet.org) a été créé. Le CEFIC (conseil européen de l'industrie chimique, www.cefic.org) a lancé le programme LRI (long range research initiative, www.cefic-lri.org) qui a pour but de coordonner la recherche publique et privée au niveau européen, de même que l'ECPI (European council for plasticisers and intermediates) finance des projets visant à élucider les effets des phtalates sur la santé animale et humaine. En France, le Ministère de l'écologie et du développement durable dispose d'un certain nombre de conseils et comités nationaux, dont le comité de prévention et de précaution (CPP) (http://www.ecologie.gouv.fr/rubrique.php3?id_rubrique $=444$ ) qui publie des rapports réguliers (http://www. ecologie.gouv.fr/article.php3?id_article=1827). Ce même ministère a aussi lancé en 2005 un programme national de recherche sur des perturbateurs endocriniens (PNRPE; http://www.ecrin.asso.free.fr/pdf/prog/apr2005pnrpe.pdf). Enfin, le Ministère de l'Éducation nationale, de l'enseignement supérieur et de la recherche (http://www.recherche. gouv.fr/rapport/santetravail/1.1contaminantsmilieuxexpos. pdf) a inscrit la problématique «Atteintes de la reproduction » dans le Plan National Santé Environnement et le Plan Santé au Travail. Le groupe ECRIN articule des réflexions de l'industrie publique et privée et de différents acteurs de la recherche et les pouvoirs publics (www.ecrin.asso.fr). Les principaux points prioritaires qui méritent de plus amples recherches figurent dans le rapport récent publié par le Ministère de l'Éducation Nationale, de l'Enseignement Supérieur et de la Recherche (http://www.recherche.gouv.fr/rapport/santetravail/l.1 contaminantsmilieuxexpos.pdf). $\diamond$

\section{SUMMARY}

The concept of endocrine disruption and human health

In Europe, endocrine disruptors (EDs) have been defined as substances foreign to the body that have deleterious effects on the individuals or their descendants, due to changes in endocrine function. In the United States, EDs have been described as exogenous agents that interfere with the production, release, transport, metabolism, binding, action or elimination of the natural ligands responsible for maintaining homeostasis and regulating body development. These two definitions are complementary, but both indicate that the effects induced by EDs probably involve mechanisms relating in some way to hormonal homeostasis and action. EDs are generally described as substances with anti-oestrogenic, oestrogenic, anti-androgenic or androgenic effects. More 
recently, other targets have been evidenced such as the thyroid and immune system. Many different $\varepsilon D$ s are present in the various compartments of the environment (air, water and land) and in foods (of plant and animal origin). They may originate from food packaging, combustion products, plant health treatments, detergents and the chemical industry in general. In addition to the potential effects of these compounds on adults, the sensitivity of embryos and fetuses to many of the xenobiotic compounds likely to cross the placenta has raised considerable concern and led to major research efforts. With the exception of the clearly established links between diethylstilbestrol, reproductive health abnormalities and cancers, very little is known for certain about the effects of $\varepsilon D s$ on human health. Given the lack of available data, current concerns about the possible involvement of $\varepsilon D s$ in the increase in the incidence of breast cancer, and possibly of endometriosis and early puberty in girls, remain hypothetical. Conversely, the deterioration in male reproductive health is at the heart of preoccupations and progress in analyses of the relationship between $\varepsilon D$ s and human health. This literature review aims to describe the current state of knowledge about endocrine disruption, focusing in particular on the problem of food contaminants. $\diamond$

\section{RÉFÉRENCES}

1. Kavlock RJ, Daston GP, DeRosa C, et al. Research needs for the risk assessment of health and environmental effects of endocrine disruptors: a report of the U.S. EPA-sponsored workshop. Environ Health Perspect $1996 ; 104$ (suppl 4) : 715-40.

2. Toppari J, Larsen JC, Christiansen P, et al. Male reproductive health and environmental xenœstrogens. Environ Health Perspect 1996 ; 104 (suppl 4) : 741-803.

3. Jégou B, Auger J, Multigner L, et al. The saga of the sperm count decrease in humans and wild and farm animals. In: Gagnon C, ed. The male gamete: from basic sciences to clinical applications. Vienna, IL, USA : Cache River Press 1999: 445-54.

4. Eustache F, Auger J, Cabrol D, Jouannet P. Are volunteers delivering semen samples in fertility studies a biased population? Hum Reprod 2004 ; $19: 2831-7$.

5. Auger J, Kunstmann JM, Czyclik F, Jouannet P. Decline in semen quality among fertile men in Paris during the past 20 years. N EnglJ Med 1995 ; $332: 281-5$.

6. Swan SH, Main KM, Liu F, et al. Decrease in anogenital distance among male infants with prenatal phthalate exposure. Environ Health Perspect 2005 ; 113 : 1056-61.

7. Skakkebaek NE, Rajpert-De Meyts $\varepsilon$, Main KM. Testicular dysgenesis syndrome: an increasingly common developmental disorder with environmental aspects. Hum Reprod 2001 ; 16 : 972-8.

8. Slama R, Bouyer J, Remontet L, Spira A. Epidemiology of male reproductive function: a field searching for tools. Rev Epidemiol Sante Publ $2004 ; 52: 221-42$.

9. Jensen TK, Joffe M, Scheike T, et al. Time trends in waiting time to pregnancy among Danish twins. Hum Reprod 2005; 20 : 955-64.

10. Boisen K, Chellakooty M, Schmidt I, et al. Hypospadias in a cohort of 1072 Danish newborn boys: prevalence and relationship to placental weight, anthropometrical measurements at birth, and reproductive hormone levels at 3 months of age. J Clin Endocrinol Metab 2005; $90: 4041-6$.

11. Cheek OA, Kow K, Chen J, McLachlan JA. Potential mechanisms of thyroid disruption in humans : interaction of organochlorine compounds with thyroid receptor, transthyretin and thyroid-binding globulin. Environ. Health Perspect $1999 ; 107: 273-8$.
12. Arcaro KF, Yi L, Seegal RF, et al. 2,2', 6, 6'-tetrachlorobiphenyl is œstrogenic in vitro and in vivo. J Cell Biochem 1999; $72: 94-102$.

13. Kester MHA, Bulduk S, Tibboel D, et al. Potent inhibition of œstrogen sulfotransferase by hydroxylated PCB metabolites: a novel pathway explaining the œstrogenic activity of PCBs. Endocrinology $2000 ; 141: 1897-1900$.

14. Mocarelli P, Gerthoux PM, Ferrari $\varepsilon$, et al. Paternal concentrations of dioxin and sex ratio of offspring. Lancet $2000 ; 355: 1858-63$.

15. Meerts IA, Letcher RJ, Hoving $S$, et al. In vitro estrogenicity of polybrominated diphenyl ethers, hydroxylated PDBEs, and polybrominated bisphenol A compounds. Environ Health Perspect $2001 ; 109: 399-407$.

16. Acevedo R, Parnell PG, Villanueva H, et al. The contribution of hepatic steroid metabolism to serum estradiol concentrations in nonylphenol treated MMTVneu mice and its potential effects on breast cancer incidence and latency. J Appl Toxicol $2005 ; 25$ : 339-53.

17. Calafat AM, Kuklenyik Z, Reidy JA, et al. Urinary concentrations of bisphenol A and 4nonylphenol in a human reference population. Environ Health Perspect $2005 ; 113: 391-5$.

18. Markey CM, Luque EH, Munoz de Toro MM, et al. In utero exposure to bisphenol A alters the development and tissue organization of the mouse mammary gland. Biol Reprod 2001 ; $65: 1215-23$.

19. Timms BG, Howdeshell KL, Barton L, et al. CEstrogenic chemicals in plastic and oral contraceptives disrupt development of the fetal mouse prostate and urethra. Proc Natl Acad Sci USA 2005 ; 102 : 7014-9.

20. Zalko D, Soto AM, Dolo L, et al. Biotransformations of bisphenol $A$ in a mammalian model: answers and new questions raised by low-dose metabolic fate studies in pregnant CDI mice. Environ Health Perspect $2003 ; 111$ : 309-19.

21. Saillenfait AM, Laudet-Lesbert A. Phtalates. EMC-Toxicologie-Pathologie, Elsevier Paris, France $2005 ; 2: 1-13$.

22. Foster PM. Disruption of reproductive development in male rat offspring following in utero exposure to phthalate esters. Int J Androl 2006; $29: 140-7$.

23. Mahood IK, McKinnell C, Walker M, et al. Cellular origins of testicular dysgenesis in rats exposed in utero to di(n-butyl) phthalate. Int J Androl $2006 ; 29$ : 148-54.

24. Zacharewski TR, Meek MD, Clemons JH, et al. Examination of the in vitro and in œstrogenic activities of eight commercial phthalate esters. Toxicol Sci $1998 ; 46: 282-93$.

25. Colon I, Caro D, Bourdony CJ, Rosario 0. Identification of phthalate esters in the serum of young Puerto Rican girls with premature breast development. Environ Health Perspect 2000 ; $108: 895-900$.

26. Taylor JR, Selhorst JB, Houff SA, Martinez AJ. Chlordecone in toxication in man. I. Clinical observations. Neurology $1978 ; 28: 626-30$.

27. Shelby MD, Newbold RR, Tully DB, et al. Assessing environmental chemicals for œstrogenicity using a combination of in vitro and in vivo assays. Environ Health Perspect 1996 ; $104: 1296-300$.

28. Monosson $\varepsilon$, Kelce WR, Lambright C, et al. Peripubertal exposure to the antiandrogenic fungicide, vinclozolin, delays puberty, inhibits the development of androgen-dependent tissues, and alters androgen receptor function in the male. Toxicol Ind Health 1999 ; $15: 65-79$.

29. Anway MD, Cupp AS, Uzumcu M, Skinner MK. Epigenetic transgenerational actions of endocrine disruptors and male fertility. Science $2005 ; 308$ : 1466-9.

30. Barnes S. Evolution of the health benefits of soy isoflavones. Proc Soc Exp Biol Med 1998; $217: 386-92$

31. Bennetau-Pelissero C, Latonnelle K, Séqueira A, Lamothe V. Phytœstrogens, endocrine disrupters from food. Analusis $2000 ; 28: 763-75$.

32. Murphy PA, Song T, Buseman G, et al. Isoflavones in retail and institutional soy foods. J Agric Food Chem 1999; $47: 2697-704$.

33. Setchell KDR, Zimmer-Nechemias L, Cai J, Heubi JE. Isoflavone content of infant formulas and the metabolic fate of these phytœstrogens in early life. Am J Clin Nutr 1998; 68 (suppl) : S1453-61.

34. Hilakivi-Clarke L, Cho $\varepsilon$, Onojafe I, et al. Maternal exposure to genistein during pregnancy increases carcinogen-induced mammary tumoregenesis in female rat offspring. Oncol Rep $1999 ; 6: 1089-95$.

35. Newbold RR, Padilla Banks $\varepsilon$, Bullock B, Jefferson WN. Uterine adenocarcinoma in mice treated neonatally with genistein. Cancer Res $2001 ; 61: 4325-8$.

36. Drash AL, Kramer MS, Swanson J, Udall JN. Infant breeding practices and their possible relationship to the etiology of diabetes mellitus. Pediatrics $1994 ; 94: 752-4$.

37. North K, Golding J. A maternal vegetarian diet in pregnancy is associated with hypospadias. BrJ Urol Int $2000 ; 85$ : 107-13.

38. Europa-environment. How the European commission uses the precautionary principle to tackle endocrine disrupters. Bruxelles : UE, 2005. http://europa.eu.int/comm/environment/ endocrine/.

\section{TIRÉS À PART}

B. Jégou 